\title{
Model-free computation of ultra-short-term prediction intervals of solar irradiance
}

\author{
D. Torregrossa*, J.-Y. Le Boudec, M. Paolone \\ Swiss Federal Institute of Technology in Lausanne, Switzerland
}

Received 2 September 2015; received in revised form 16 November 2015; accepted 17 November 2015

Communicated by: Associate Editor Mario A Medina

\begin{abstract}
We propose an ultra-short-term dynamic interval predictor (DIP) of solar irradiance. Our DIP relies on experimentally observed correlations between the derivative of the solar irradiance and the forecast error in the next time-step. The main originalities of this DIP are (i) its independence from the method used for the point forecast of solar irradiance, (ii) its independence from the error distribution of the point-forecast method. We compare the DIP with the most common prediction interval methods. By using significant data set covering several months of experimental observations, we have observed higher accuracy and lower width of the prediction intervals of the proposed DIP.
\end{abstract}

(c) 2015 Elsevier Ltd. All rights reserved.

Keywords: Forecast; Ultra short-term; Solar irradiance; Prediction intervals

\section{Introduction}

Today's trend of vast connections of distributed generation in low- and medium-voltage power networks accounts for quality-of-supply of electrical distribution grids in a way that, in several countries, operational constraints are already attained. Additionally, it is necessary that their active contribution be quantified in real-time and, eventually, controlled. In this respect, one of the main concerns of distribution network operators refers to the definition of optimal control-schemes in which the high volatility of renewable-energy resources (RERs) can be accounted for. The choice of the forecast time window is extremely important and it is highly correlated to the design of real-time control of RERs in order to provide grid primaryancillary services (e.g., Song et al., 2013; Vrakopoulou

\footnotetext{
* Corresponding author. Tel.: +41 787209227; fax: +41 (0) 216934662 .

E-mail address: dimitri.torregrossa@epfl.ch (D. Torregrossa).
}

et al., 2013; Heniche et al., 2013). Several control strategies have been proposed (database model in Song et al. (2013), stochastic optimization in Vrakopoulou et al. (2013), multiagents in Heniche et al. (2013)) to define dedicated realtime energy-management systems and, in some cases, the concept of real-time control is associated with time dynamics below $1 \mathrm{~s}$ (Heniche et al., 2013).

More specifically, the authors of Bernstein et al. (2015), Reyes Chamorro et al. (2015) recently proposed a solution to the challenging problem of controlling a distribution network in real-time by using explicit power setpoints. In this framework the resources can advertise their current internal needs and power availability by simple messages in order to enable a grid controller to maintain the state of the system within secure limits. The framework, called Commelec, is designed to be robust (i.e., it avoids the problems inherently posed by software controllers) and scalable (i.e., it easily adapts to grids of any size and complexity). It is based on software agents, that are responsible for 
resources/subsystems (Resource Agents) or entire grids (Grid Agents) and they communicate using a simple yet powerful protocol with a refresh rate of around $100 \mathrm{~ms}$. A detailed description of the proposed framework is given in Section 2.

In this context, the real-time control can be considerably improved if the Grid Agents are able to bound the uncertainty of power injections, due to stochastic sources, at a horizon of one or a few control cycles (fraction of a second). For systems with photovoltaic (PV) panels, it is worth observing that the solar irradiance has an extreme volatility in time scales below a second. ${ }^{1}$ It is thus interesting to find ultra-short-term forecast bounds for the solar irradiance of PV panels, and such is our goal in this paper.

The available literature on prediction intervals for PV energy-conversion systems is characterized by the following four main limitations (Singh et al., 2013; Kardakos et al., 2013; Trapero et al., 2014; Lorenz et al., 2009; Marquez and Coimbra, 2011; Bacher et al., 2009; Segura and Vercher, 2001): (i) absence of methods proposing prediction intervals targeting the time scale of seconds or sub-seconds; (ii) absence of methods proposing prediction intervals able to track the highly-dynamic volatility of the solar irradiance; (iii) absence of methods able to account for distributions of the point-forecast errors other than Gaussian; (iv) strong dependency of the prediction interval with the specific method used for the point forecast computation. To the best of our knowledge, the only works that are independent of the point-forecast method are (Wan et al., 2014; Pinson and Tastu, 2014). Machine-learning methods capable of quantifying uncertainty bounds of point forecasts are presented in Wan et al. (2014), Pinson and Tastu (2014).

In this paper we propose a model-free prediction interval of the solar irradiance. The method, henceforth called the dynamic interval predictor (DIP), is able to estimate the magnitude of the prediction intervals by assessing the correlations between the measurements of the derivative of solar irradiance and the point-forecast error in the next forecasting time-step.

With respect to the above-listed drawbacks of traditional prediction intervals, the DIP exhibits the following characteristics: (i) the prediction intervals are computed within a time scale ranging from $250 \mathrm{~ms}$ up to $750 \mathrm{~ms}$; (ii) it does not depend directly on the method used for the point forecast; (iii) it is able to track high dynamics of the solar irradiance and (iv) it is capable of selfimproving its performances during its use because it is able to correct the magnitude of the prediction intervals for future computations.

The paper is structured as follows. The Commelec framework, for which the proposed DPI has been deployed, is described in Section 2. A brief summary of the different existing methods for prediction intervals is

\footnotetext{
${ }^{1}$ An experimental quantification of the sub-second PV volatility is given in Section 3.
}

reported in Section 3. In order to highlight the need of ultra-short-term forecast, experimental evidences of subsecond solar dynamics are illustrated in Section 4. In the same section, by using experimental data, the existing correlations between the derivative of solar irradiance and the point-forecast error in the next forecasting time-step have been analyzed. The proposed DIP is described in detail in Section 5. The robustness of the DIP, and its comparison with the other commonly used prediction intervals methods, are illustrated in Section 6. In particular, since the available literature on point forecast computation contains a considerable amount of works based on heuristic technique (Mellit and Pavan, 2010; Mellit and Kalogirou, 2008; Sfetsos and Coonick, 2000; Behrang et al., 2010), Section 6 also assesses the performances of the proposed DPI coupled with an ANFIS (adaptive neuro-fuzzy inference system) point forecast model. The main findings of the work and its applicability are summarized in Section 7.

\section{The Commelec control framework}

In the Commelec framework, a software agent is associated with a resource (henceforth called "Resource Agent", RA), or an entire system, including a grid and/or a number of devices (henceforth called "Grid Agent", GA). There is a well-defined relationship between the agents, which follows from the tree structure of the distribution networks. An example of agents relationship is shown in Fig. 1 where GA is in charge of controlling RAs $A_{1}, \ldots, A_{N}$, who are responsible for subsystems $S_{1}, \ldots, \mathrm{S}_{N}$.

Each Resource Agent advertises its internal state to its Grid Agent using the following three elements. (1) The $P Q$ profile $A$ is the region in the $(P, Q)$ plane (for active and reactive power) that the subsystem under the control of this Resource Agent can deploy. (2) The virtual cost $C$ is a function, that defined for every $(P, Q)$ in the $P Q$ profile, returns a number $C(P, Q)$ interpreted as the willingness of this subsystem to apply a requested power setpoint $(P, Q)$. It is virtual in the sense that it is not directly related to a monetary value. (3) The belief function BF returns the set of all possible (actual) setpoints so that this subsytem might in reality implement, when instructed to implement a target setpoint. This accounts for the uncertainty in a subsystem operation. In particular, highly controllable subsystems are expected to have ideal beliefs, namely

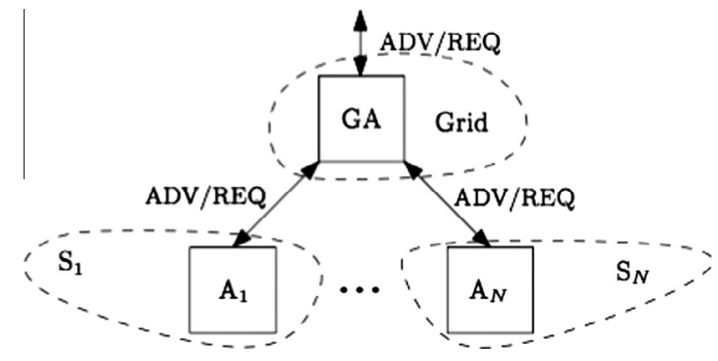

Fig. 1. A general scheme for showing Commelec agents interactions. 
$B F(P, Q)=\{(P, Q)\}$. For subsystems such as $\mathrm{PV} /$ wind farms, or loads, the belief function returns larger sets, to account for their volatility. It is important to observe that these three elements (i) are the only information needed for real-time control in the Commelec framework and (ii) hide the internal state of the resource.

\section{State of the art: prediction intervals}

The main approaches, presented in the literature, to the problem of the PV short-term forecasting are summarized by Singh et al. (2013), Kardakos et al. (2013), Trapero et al. (2014), Lorenz et al. (2009), Marquez and Coimbra (2011), Bacher et al. (2009). The authors of Singh et al. (2013) propose an adaptive-neuro-fuzzy inference (ANFIS) to predict the PV power output in the time horizon of onehour ahead. The input quantities of the forecasting tool are solar irradiance, ambient temperature and wind velocity. The committed error for a one-hour ahead prediction is in the order of 9.6\%. In Kardakos et al. (2013) the authors propose also an auto regressive integral moving average (ARIMA) model to predict a day-ahead PV power production. The analyzed data cover one year and the average of the relative root mean square error (RMSE) of the proposed forecast method is equal to $11 \%$. In Trapero et al. (2014), the authors used an auto regressive moving average (ARMA) model to predict one hour-ahead the solar irradiance by taking into account in their analysis clear and cloudy days. In Trapero et al. (2014) the authors use a simple exponential-smoothing algorithm to forecast the onehour ahead solar irradiance.

Concerning the prediction intervals, a common hypothesis adopted in the literature (e.g., Lorenz et al., 2009; Marquez and Coimbra, 2011) is to assume a normal distribution of the forecast errors. The magnitude of the prediction intervals are usually estimated as multiples of the standard deviation of the forecast solar-irradiance associated with a given confidence level. In general, the prediction intervals are computed only for one-hour ahead predictions and, as illustrated in the works mentioned above, the width of those intervals is large. It ranges between $14 \%$ and $80 \%$ of the point-forecast value.

Bacher et al. (2009) propose a prediction-intervals computation by using quantile regression. Their proposed method is suitable for online forecasting. However, the magnitude of the prediction intervals could be quite important (i.e., in the range of $80 \%$ of the point forecast).

We summarize below the most common methods presented in the literature for the prediction of intervals of random variables; these methods could be adopted to the case of PV production. The two quantities that are generally forecasted are the PV AC output power and the solar irradiance. In our work we focus only on the prediction intervals for solar irradiance.

The purpose of any prediction interval is to satisfy, at any forward step $t_{k}$, the following equation with a certain confidence $\eta$.

$$
\widehat{P}_{\mathrm{IRR}}^{k}-W_{B, \mathrm{LOW}}^{k} \leqslant P_{\mathrm{IRR}}^{k} \leqslant \widehat{P}_{\mathrm{IRR}}^{k}+W_{B, \mathrm{UPP}}^{k}
$$

$$
\text { with }
$$

- $\widehat{P}_{\mathrm{IRR}}^{k}$ : predicted solar irradiance at time-step $k$.

- $P_{\mathrm{IRR}}^{k}$ : measured solar irradiance at time-step $k$.

- $W_{B, \mathrm{LOW}}^{k}$ : lower-bound of the prediction interval at timestep $k$.

- $W_{B, \mathrm{UPP}}^{k}$ : upper-bound of the prediction interval at timestep $k$.

There are several methods for forecasting the behavior of stochastic physical phenomena. A thorough study of the literature on this topic shows that there are two main types of models: (i) ARMA/ARIMA models and (ii) adaptive neural-fuzzy inference system. The first category can be used in presence of a stochastic process with a clear autocorrelation structure; it has a clear method for computing the prediction intervals. The additive Holt-Winters method belongs to this category; it is often used as it has very few parameters and is robust. The persistent predictor, which uses as prediction for the next time-step the most recent measured value, also belongs to this category and is often used as a simple benchmark. The second category can be adapted to different domains because they learn, in a certain way, the behavior of the variable to be predicted. However, this category does not give any indications about the prediction-interval computation.

For the ARMA/ARIMA models with Gaussian innovation, the upper and lower bound of the predicted intervals can be evaluated as follows (Le Boudec, 2010):

$W_{B, \mathrm{LOW}}^{k+1}=W_{B, \mathrm{UPP}}^{k+1}=\chi \sqrt{\sigma_{k}}$

where $\chi$ is a coefficient depending on the required confidence level for the prediction interval and $\sigma_{k}$ is the variance of the prediction error, computed from the model and the residuals.

To the best of our knowledge, all the works presented in the domain of PV forecasting assume a Gaussian innovation. In this respect, in this paper we also consider nonGaussian innovations. First, the generalized autoregressive conditional heteroskedasticity $(\mathrm{GARCH})$ model can be used to model the noise. The main characteristic is that enables the prediction of its variance, at time $k$, by using the following equation:

$$
\begin{gathered}
\sigma_{t}^{2}=\omega+\sum_{1}^{q} \phi_{i} \varepsilon_{t-i}^{2}+\sum_{1}^{p} \psi_{i} \sigma_{t-i}^{2} \\
\omega, \varphi_{i}, \psi_{i}>0 \\
\varphi_{i}+\psi_{i}<1
\end{gathered}
$$

where

- $\varepsilon$ is a white noise.

- $\omega, \phi, \psi$ are coefficients to be determined by an optimization problem, described in details in Brockwell and Davis (2002). 
Once the variance at time $t=t_{k}$, has been predicted, the upper and lower bounds of the prediction interval are also computed with Eq. (2).

Another method for account for non-Gaussian innovation is the Bootstrap (Le Boudec, 2010). In this case, it is necessary to evaluate the error distribution. Once the value of the confidence level $\eta$ has been defined, it is possible to detect, in the Bootstrap distribution two values of error associated with the quantiles $\frac{1-\eta}{2}$ and $\frac{1+\eta}{2}$ (these two values are defined as $\bar{E}_{\mathrm{BTS}}^{\min }$ and $\bar{E}_{\mathrm{BTS}}^{\max }$ ). Consequently, the computation of the prediction intervals can be performed, for future predictions, as follows:

$$
\begin{aligned}
& W_{B, \mathrm{LOW}}=\bar{E}_{\mathrm{BTS}}^{\min }(\eta) \\
& W_{B, \mathrm{UPP}}=\bar{E}_{\mathrm{BTS}}^{\max }(\eta)
\end{aligned}
$$

It is important to underline that the width of the prediction intervals computed with (3) and (4) is independent of the dynamics of the solar irradiance.

\section{Experimental evidences of correlations between solar irradiance dynamics and prediction errors}

By means of experimental evidences in this section we assess the correlations between the solar irradiance and the prediction errors in a forecasting-time window of the order of sub-seconds. In this respect, we first describe the adopted experimental setup and, then, we numerically analyze the above-mentioned correlations.

\subsection{Experimental setup}

The experimental setup used for the experimental characterization of the dynamics associated with the solar irradiance includes a Phono Solar $240 \mathrm{~W}$ PV module (PS240P-20/U) and an Enphase M215 monophase microinverter $(\mu$-inv). The $\mu$-inv is a grid-tie converter that always uses the maximum power point-tracking (MPPT) method. The sensors deployed in this experimental setup are: (i) an Apogee SP-230 pyrometer for the irradiance in the same plane of the PV module (the bandwidth of the sensor is $1 \mathrm{kHz}$ ), (ii) two IST AG TSic TO92 temperature sensors for the module's temperature (one on the surface and one behind the PV); (iii) two current sensors LEM LAH 25-NP for $\mathrm{DC}$ and $\mathrm{AC}$; and (iv) two voltage sensors LEM LV 20-P for DC and AC voltages. The system is located at the following GPS location: 46.518397-N, 6.565229-E.

\subsection{Observed irradiance dynamics}

We used the above-described experimental setup to collect data for a period of one year. In order to evaluate the dynamic of $P_{\text {IRR }}$, we computed its discrete time derivative at each time-step forecast. Based on this one-year analysis, $P_{I R R}$ reached a maximum of $1400 \mathrm{~W} / \mathrm{m}^{2}$ and its derivative reached a maximum of $60 \mathrm{~W} / \mathrm{s} / \mathrm{m}^{2}$. As we focus on ultrashort-term prediction intervals of the solar irradiance, it is worth observing that its variations are only associated with clouds passing and not with clear sky dynamics. In this respect, Fig. 2 illustrates a typical time evolution of the solar irradiance during cloud passing at the measurement location. In this figure we observe time derivatives of the solar irradiance of about $40 \mathrm{~W} / \mathrm{s} / \mathrm{m}^{2}$.

As already stated in the introduction, our DIP is based on the investigation of the correlation between the forecast relative-error $e_{\mathrm{IRR}}^{k+1}$ at time $k+1$ and the derivative of the solar-irradiance time at time $k$, defined by $\dot{P}_{\mathrm{IRR}}^{k}=P_{\mathrm{IRR}}^{k}-P_{\mathrm{IRR}}^{k-1}$. In this respect, in Fig. 2 we illustrate the cumulative-distribution function of the error associated with different values of the derivative when the point forecast is obtained with the double-exponential Holt Winters method. We can observe that the conditional distribution of the forecast error, given the value $\dot{P}_{\text {IRR }}^{k}$ of the derivative of the irradiance in the previous time-step, strongly depends on $\dot{P}_{\mathrm{IRR}}^{k}$. We will use this dependency to derive our DIP.

The results of Fig. 3 show that it is possible to statistically quantify the error made by the forecasting process as a function of the irradiance time derivative, by

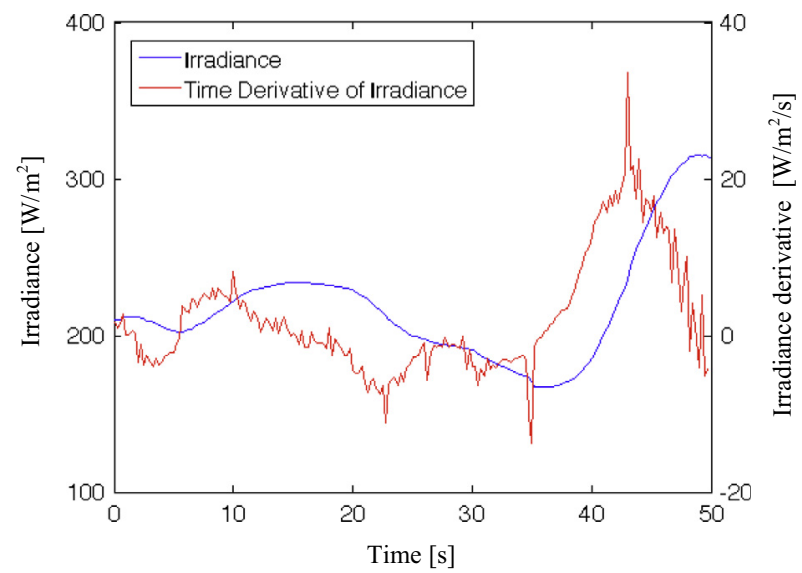

Fig. 2. Example of the highest $P_{\mathrm{IRR}}$ dynamics measured on 24th Mars, 2014 at location $46.518397-\mathrm{N}, 6.565229-\mathrm{E}$.

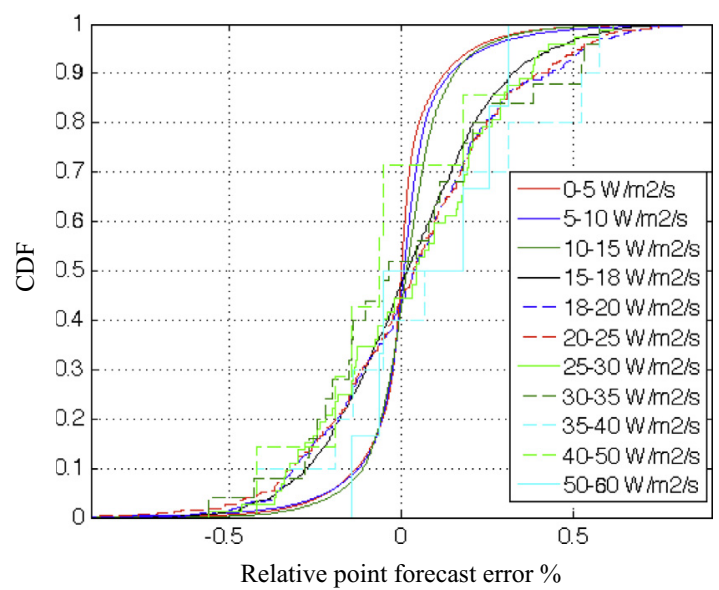

Fig. 3. Cumulative conditional distribution function of the relative forecast error $e_{\mathrm{IRR}}^{k+1}$ given the derivative of the irradiance. 
estimating the conditional distribution of the prediction error given the derivative of the irradiance. This observation is the starting point of the DIP method, presented in the next section.

\section{The proposed dynamic-interval predictor}

As anticipated in the introduction, we target a DIP that is independent of the point-forecast method and of the point-forecast error distribution. In this respect, in this sub-section we describe the algorithm for the DIP without adopting any a priori assumption on a specific pointforecast method.

\subsection{Overview on the proposed DIP}

In this sub section we summarize the steps of the algorithm for computing the lower and upper bounds of the irradiance prediction, $\left(W_{B, \mathrm{LOW}}^{k+1}\right)$ and $\left(W_{B, \mathrm{UPP}}^{k+1}\right)$ respectively.

(1) Irradiance measurement and computation of its derivative $\dot{P}_{\mathrm{IRR}}^{k}$.

(2) Prediction of the irradiance measurement at the next time-step $\widehat{P}_{\text {IRR }}^{k+1}$.

(3) Discretization of $\dot{P}_{\mathrm{IRR}}^{k}$ and $e_{\mathrm{IRR}}^{k+1}$.

(4) Computation of the initial value of the conditional distribution of $e_{\mathrm{IRR}}^{k+1}$ given $\dot{P}_{\mathrm{IRR}}^{k}$; the result is stored in the matrix $\mathbf{R}$.

(5) Computation of the upper and lower bound intervals $W_{B, \mathrm{LOW}}^{k+1}$ and $W_{B, \mathrm{UPP}}^{k+1}$ via the correlations assessed at point 3.

(6) Matrix $\mathbf{R}$ update.

In what follows a comprehensive description of the above-listed steps is given.

\subsection{Conditional distribution matrix $\boldsymbol{R}$}

We store the empirical conditional distribution of the forecast error, given the derivative of the irradiance in a matrix $\mathbf{R}$. Formally, $\mathbf{R}(n, m)$ is equal to the probability that the next forecast error is $e(n)$ given the derivative of the irradiance $\dot{p}(m)$, where $e(n)$ is the $n$th discretized value of errors and $\dot{p}(m)$ is the $m$ th discretized value of derivatives.

The key idea of our DIP is to use the correlations defined by matrix $\mathbf{R}$ to compute the prediction intervals, as explained in the next sub-section.

\subsection{Computation of the prediction intervals width}

The process for the computation of the prediction interval is the following:

1. Define the required value of the confidence level $\eta$.

2. At the generic time-step $t_{k}$, calculate $\dot{P}_{\mathrm{IRR}}^{k}$ (from the experimental observation)
3. Find the element $\dot{p}(m)$ that is the closest to $\dot{P}_{\mathrm{IRR}}^{k}$.

4. Compute the $\frac{1+\eta}{2}$ and $\frac{1-\eta}{2}$ quantiles of the distribution of errors that corresponds to the column $m$ of matrix R. This is done as follows. We interpret column $m$ of matrix $\mathbf{R}$ as the histogram of a probability distribution that has a continuous cumulative distribution function $F()$. The matrix $\mathbf{R}$ gives the values of at the points $e(n)$ by the formula :

$F(e(n))=\sum_{n^{\prime}=1}^{n} R\left(n^{\prime}, m\right)$

and the value of $F(e)$ at an arbitrary $e$, which is not one of the, $e(n)$ is obtained by linear interpolation. The quantiles $e_{\mathrm{IRR}}^{\mathrm{UPP}}$ and $e_{\mathrm{IRR}}^{\mathrm{LOW}}$ are obtained by the conditions $F\left(e_{\mathrm{IRR}}^{\mathrm{UPP}}\right)=\frac{1+\eta}{2}$ and $F\left(e_{\mathrm{IRR}}^{\mathrm{LOW}}\right)=\frac{1-\eta}{2}$.

The prediction interval widths are obtained by

$$
\begin{aligned}
& W_{B, \mathrm{UPP}}^{k+1}=e_{\mathrm{IRR}}^{\mathrm{UPP}} \cdot \widehat{P}_{\mathrm{IRR}}^{k+1} \\
& W_{B, \mathrm{LOW}}^{k+1}=e_{\mathrm{IRR}}^{\mathrm{LOW}} \cdot \widehat{P}_{\mathrm{IRR}}^{k+1}
\end{aligned}
$$

Consequently, the width of the DIP depends on the required confidence level and a brief analysis of this dependence is illustrated in Table 2.

\subsection{Update of the conditional distribution matrix}

A main advantage of the proposed DIP is its capability to take into account the evolution of the dynamics associated with the solar irradiance. As explained in the previous sub-section, the prediction-interval computation changes at each time-step and is based on the knowledge of the conditional distribution matrix $\mathbf{R}$. In our work, we investigate three different ways to update this conditional distribution matrix. These three methods are:

- Step-by-step update: the $\mathbf{R}$ update is performed at each time-step and it is fed by the raw sampled data of the irradiance (method \#1).

- Batch update: the update takes into account a larger amount of points from the beginning of the measurement, without any weight for the last correlations between $\dot{P}_{\mathrm{IRR}}^{k}$ and $e_{\mathrm{IRR}}^{k+1}$ (method \#2).

- Weighted step-by-step: this method puts more weight on the recent correlations (method \#3).

We can observe that methods \#1 and \#2 are similar, whilst \#3 gives a way to gradually age out the old "learnt" dynamics.

The step-by-step update method \#1 is based on the following equations. Let assume that, at time $t_{k}$, we make a new observation, and let $e\left(n_{0}\right)$ and $\dot{p}\left(m_{0}\right)$ be the discretized values of the error $e_{\mathrm{IRR}}^{k+1}$ and the derivative $\dot{P}_{\mathrm{IRR}}^{k}$. The update of the equations for matrix $\mathbf{R}^{\mathrm{NEW}}$ is then as follows: 


$$
\begin{aligned}
& \mathbf{R}^{\mathrm{NEW}}\left(n_{0}, m_{0}\right)=\frac{N^{\mathrm{OLD}}\left(n_{0}, m_{0}\right)+1}{N^{\mathrm{OLD}}\left(m_{0}\right)+1} \\
& \mathbf{R}^{\mathrm{NEW}}\left(n, m_{0}\right)=\frac{N^{\mathrm{OLD}}\left(n, m_{0}\right)}{N^{\mathrm{OLD}}\left(m_{0}\right)+1}, n \neq n_{0} \\
& \mathbf{R}^{\mathrm{NEW}}(n, m)=\mathbf{R}^{\mathrm{OLD}}(n, m), n \neq n_{0}, m \neq m_{0}
\end{aligned}
$$

where

- $N^{\mathrm{OLD}}\left(m_{0}\right)$ is the number of observations having a derivative in the range of $\dot{p}\left(m_{0}\right)$.

- $N^{\mathrm{OLD}}\left(n_{0}, m_{0}\right)=\mathbf{R}^{\mathrm{OLD}}(n, m) N^{\mathrm{OLD}}\left(m_{0}\right)$.

The superscripts ${ }^{\mathrm{NEW}}$ and ${ }^{\mathrm{OLD}}$ refer to the updated and the old version of the $\mathbf{R}$ matrix, respectively.

Method \#2 uses the same set of Eq. (7), but it is not used at each time-step; instead it is applied in a batch, after a specific measurement time window. In our case, illustrated in Table 1, during the first day of measurement, the first version of $\mathbf{R}$ matrix is built and then it is updated at times 10 days, 20 days, 30 days and so on.

Method \#3 puts more weight on the last measurements. To understand how it is derived, first note that it is possible to re-write the update Eq. (7) of method \#1 as

$$
\begin{aligned}
& \mathbf{R}^{\mathrm{NEW}}\left(n_{0}, m_{0}\right)=(1-w) \mathbf{R}^{\mathrm{OLD}}\left(n_{0}, m_{0}\right)+w \\
& \mathbf{R}^{\mathrm{NEW}}\left(n, m_{0}\right)=(1-w) \mathbf{R}^{\mathrm{OLD}}\left(n, m_{0}\right), n \neq n_{0} \\
& \mathbf{R}^{\mathrm{NEW}}(n, m)=\mathbf{R}^{\mathrm{OLD}}(n, m), n \neq n_{0}, m \neq m_{0}
\end{aligned}
$$

with $w=\frac{1}{N^{\mathrm{OLD}}\left(m_{0}\right)+1}$.
In other words, Eq. (8) expresses that, with method \#1, the weight $w$ of the last correlation between $e_{\mathrm{IRR}}^{k+1}$ and $\dot{P}_{\text {IRR }}^{k}$ becomes lower at each time-step. This could involve, after a long observation time, an $\mathbf{R}$ matrix giving the same importance to all the correlations, even those observed when we computed the first version of the $\mathbf{R}$ matrix. Additionally, the last correlations that are strongly linked to the most recent behavior of the solar irradiance will not be properly taken into account. Therefore, there is the risk that old correlations no longer represent the real dynamics associated with the solar irradiance.

In contrast, with the update method \#3, the $\mathbf{R}$ matrix is updated with Eq. (8) but with a constant weight $w$. The value of $w$ controls how fast the $\mathbf{R}$ matrix ages out. To understand what a given value of $w$ represents, it is useful to consider that, roughly speaking, $1 / w$ represents the "typical time horizon" (counted in time-steps) for aging. It is worth observing that method \#3 does not require that measured data be stored because the computation of the $\mathbf{R}$ matrix inherently accounts for all the past correlations. In the next section, we show that values of $w$ that work best correspond to a time horizon of several seconds. We also compare the performance of the three update methods.

Table 1

Performances of different correlation matrix update methods.

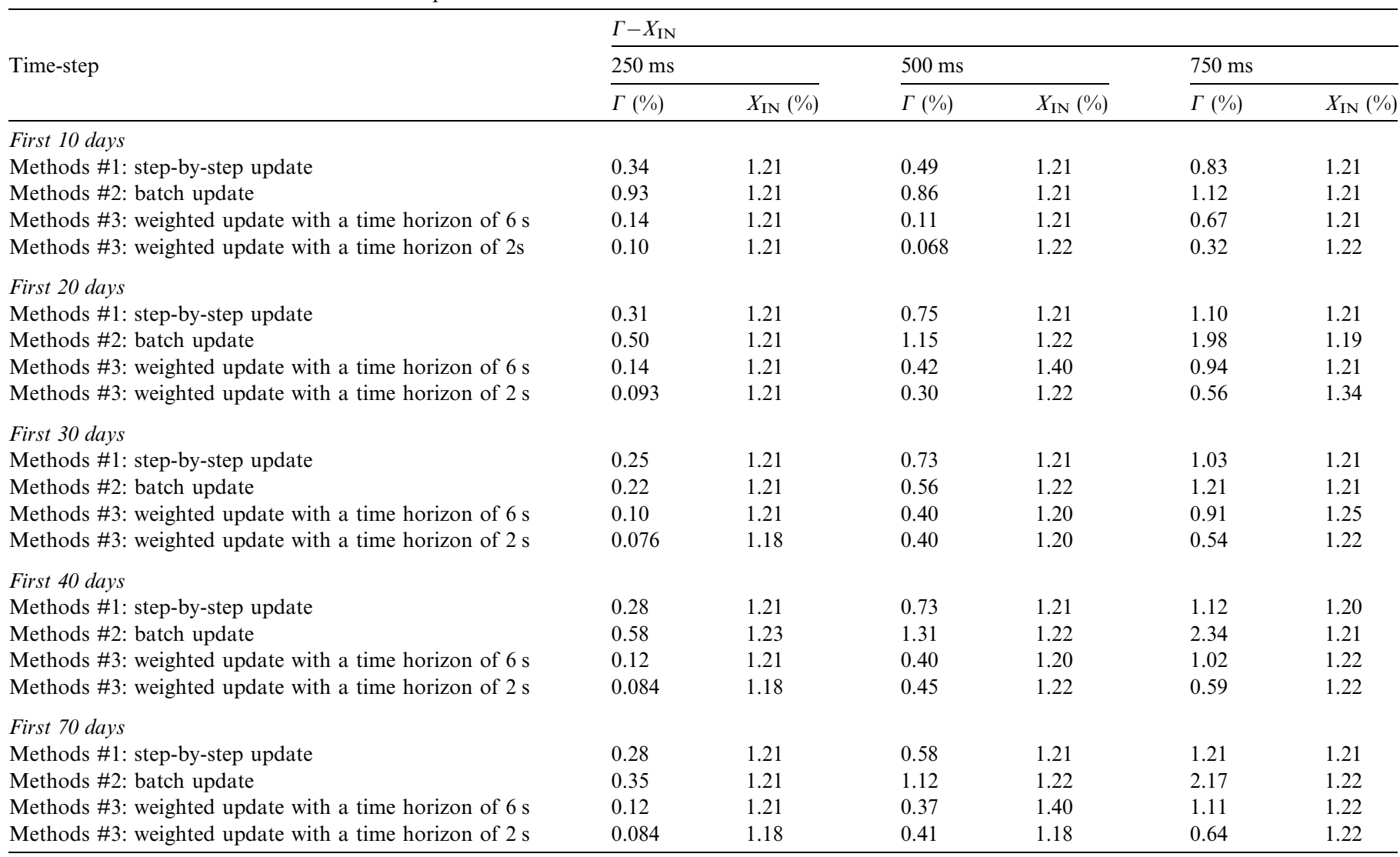


6. Experimental validation and comparison with other bound predictors

It is worth underlining that, as it can be observed from Eqs. (5)-(8), the proposed DIP does not make reference to any point-prediction method. Consequently, it is possible to assume that its description and applicability are universal.

In this section we compare the proposed DIP with the other prediction interval methods illustrated in Section 2. In this respect, it is necessary to choose a pointprediction method to which we can associate different prediction-interval computation methods and, then, compare their performances with our DIP. We chose the Holt Winters (HW) method because it relies on its simple implementation, robustness, low data-storage requirements, and straightforward automation. It also has the advantage of being able to adapt to changes in trends and seasonal

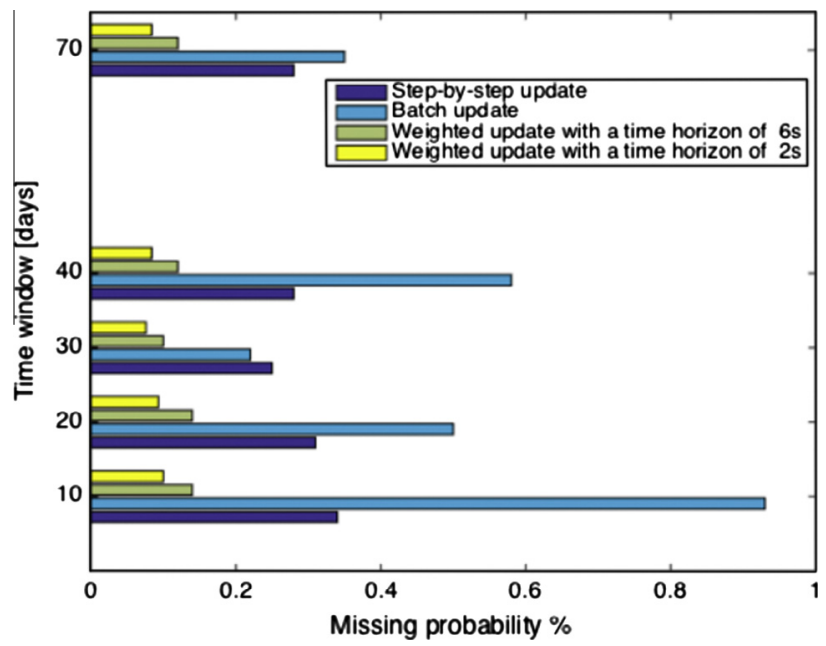

Fig. 4. Graphical comparison of the effect of different correlation matrix update methods on the missing probability (time-steps of $250 \mathrm{~ms}$ ).

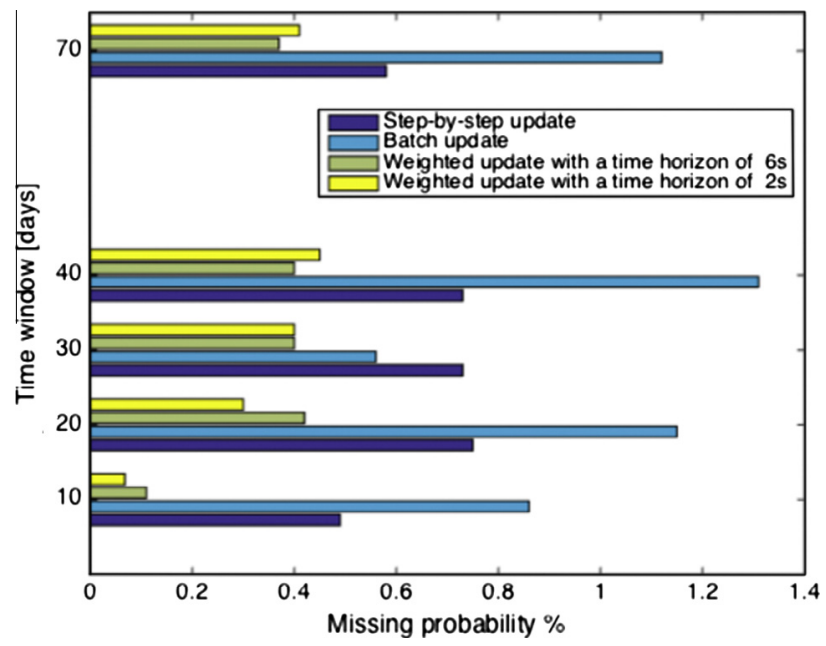

Fig. 5. Graphical comparison of the effect of different correlation matrix update methods on the missing probability (time-steps of $500 \mathrm{~ms}$ ). patterns when they occur. The complete description of the HW method can be found in Hindammn et al. (2008).

Concerning the performance assessment metrics, they are described in the next sub-section.

\subsection{Performances assessment metrics}

Any prediction-interval method can make errors. This means that, in a certain time-window, the number of measured points $(N)$ can be separated in two main categories: the points inside the prediction intervals $\left(N_{\mathrm{IN}}\right)$ and those outside ( $\left.N_{\text {OUT }}\right)$. Our first metric is the miss probability $\Gamma$ defined by

$\Gamma=\frac{N_{\text {OUT }}}{N}$

We can observe that $\Gamma$ largely depends on the width of prediction intervals, as a wider prediction interval increases the probability of obtaining points forecast satisfying

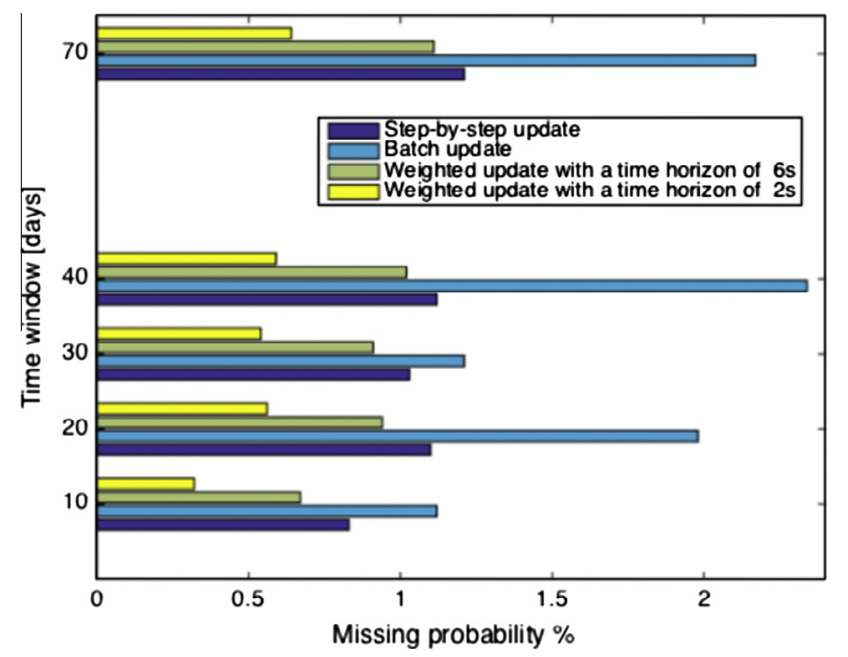

Fig. 6. Graphical comparison of the effect of different correlation matrix update methods on the missing probability (time-steps of $750 \mathrm{~ms}$ ).

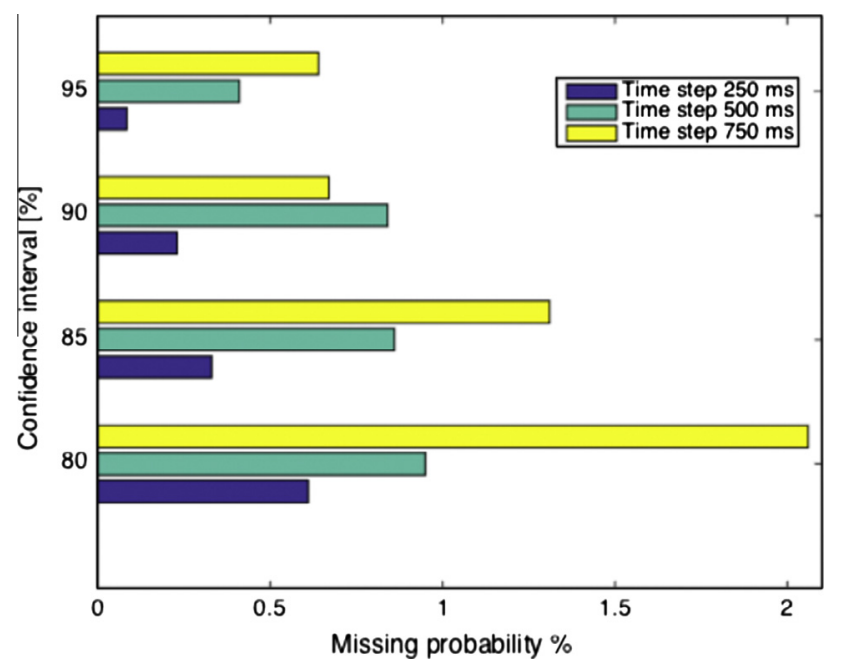

Fig. 7. Graphical comparison of the effect of different required confidence level with a weighted correlation matrix update with a time horizon of $2 \mathrm{~s}$. 
Table 2

Performance metrics with weighted update with a time horizon of $2 \mathrm{~s}$ for different confidence levels $\eta$.

\begin{tabular}{|c|c|c|c|c|c|c|}
\hline \multirow{3}{*}{ Time step (\%) } & \multicolumn{6}{|c|}{ First 70 days } \\
\hline & \multicolumn{2}{|c|}{$250 \mathrm{~ms}$} & \multicolumn{2}{|c|}{$500 \mathrm{~ms}$} & \multicolumn{2}{|c|}{$750 \mathrm{~ms}$} \\
\hline & $\Gamma(\%)$ & $X_{\mathrm{IN}}(\%)$ & $\Gamma(\%)$ & $X_{\mathrm{IN}}(\%)$ & $\Gamma(\%)$ & $X_{\mathrm{IN}}(\%)$ \\
\hline$\eta=95$ & 0.084 & 1.18 & 0.41 & 1.18 & 0.64 & 1.22 \\
\hline$\eta=90$ & 0.23 & 1.15 & 0.84 & 1.16 & 0.67 & 1.18 \\
\hline$\eta=85$ & 0.33 & 1.09 & 0.86 & 1.14 & 1.31 & 1.16 \\
\hline$\eta=80$ & 0.61 & 1.06 & 0.95 & 1.08 & 2.06 & 1.04 \\
\hline
\end{tabular}

Eq. (1). Consequently, it is extremely important to have another performance metric in order to capture the width of the prediction interval. We use the following:

$X_{\mathrm{IN}}=\frac{1}{N_{\mathrm{IN}}} \sum_{k=1}^{N} \frac{\left|W_{B, \mathrm{UPP}}^{k}-W_{B, \mathrm{LOW}}^{k}\right|}{P_{\mathrm{IRR}}^{k}} 1\{E q(1)$ holds $\}$

where the indicator function $1\{E q(1) h o l d s\}$ in the summation enables the sum only over those time instants $k$ at which (1) is satisfied. In other words, the metric $X_{\text {IN }}$ computes the mean prediction-interval, averaged over all points where the prediction interval succeeds.

\subsection{Comparison of methods for the update of the correlation matrix}

The comparison between the three matrix update methods defined in Section 5.4 in terms of $\Gamma$ and $X_{\mathrm{IN}}$ is illustrated in Table 1. In particular, three time-steps are accounted for, $250 \mathrm{~ms}, 500 \mathrm{~ms}$ and $750 \mathrm{~ms}$. The whole time-window covers 70 days of measurement during the spring season, characterized by high solar-irradiance dynamics. For each method used for the matrix update, the values of both metrics $\Gamma$ and $X_{\mathrm{IN}}$ are given. The irradiance time series is shown in Fig. 10. For the method \#2, we perform the update every 10 days, and we keep the same $\mathbf{R}$ matrix for the following 10 days. For the method \#3, we perform the update at each time-step with different values of the weight $w$; we found that the best performance is obtained with values of $w$ which correspond to a typical time horizon of a few seconds. In the following we show results for two values of $w: w_{1}$ corresponds to a typical time horizon of $6 \mathrm{~s}$ and $w_{2}$ corresponds to $2 \mathrm{~s}$. By observing Table 1, it is possible to make the following observations:

- Time-step: the most accurate results are obtained for $250 \mathrm{~ms}$ (also lower time-steps provide almost identical results).

Table 3

Comparison of different PI methods. Time step $=250 \mathrm{~ms}$.

\begin{tabular}{|c|c|c|}
\hline & $\Gamma(\%)$ & $X_{\mathrm{IN}}(\%)$ \\
\hline & \multicolumn{2}{|c|}{ First 10 days, time step $250 \mathrm{~ms}$} \\
\hline Holt Winter point forecast computation coupled with the proposed DPI with a step-by-step update & 0.34 & 1.21 \\
\hline Holt Winter point forecast computation coupled with a Gaussian error distribution & 12.47 & 2.08 \\
\hline Holt Winter point forecast computation coupled with a BootStrap error distribution & 1.40 & 7.11 \\
\hline \multirow[t]{2}{*}{ Persistent point forecast computation coupled with a Gaussian error distribution } & 66.52 & 0.23 \\
\hline & \multicolumn{2}{|c|}{ First 20 days, time step $250 \mathrm{~ms}$} \\
\hline Holt Winter point forecast computation coupled with a Garch error distribution & 21.01 & 2.25 \\
\hline Holt Winter point forecast computation coupled with a BootStrap error distribution & 31.12 & 7.89 \\
\hline \multirow[t]{2}{*}{ Persistent point forecast computation coupled with a Gaussian error distribution } & 66.38 & 0.21 \\
\hline & \multicolumn{2}{|c|}{ First 30 days, time step $250 \mathrm{~ms}$} \\
\hline Holt Winter point forecast computation coupled with the proposed DPI with a step-by-step update & 0.25 & 1.21 \\
\hline & \multicolumn{2}{|c|}{ First 40 days, time step $250 \mathrm{~ms}$} \\
\hline Holt Winter point forecast computation coupled with the proposed DPI with a step-by-step update & 0.28 & 1.21 \\
\hline Holt Winter point forecast computation coupled with a Gaussian error distribution & 12.3 & 1.81 \\
\hline Holt Winter point forecast computation coupled with a Garch error distribution & 17.61 & 2.0 \\
\hline Holt Winter point forecast computation coupled with a BootStrap error distribution & 29.58 & 7.86 \\
\hline \multirow[t]{2}{*}{ Persistent point forecast computation coupled with a Gaussian error distribution } & 71.6 & 4.77 \\
\hline & \multicolumn{2}{|c|}{ First 70 days, time step $250 \mathrm{~ms}$} \\
\hline Holt Winter point forecast computation coupled with the proposed DPI with a step-by-step update & 0.28 & 1.21 \\
\hline Holt Winter point forecast computation coupled with a Gaussian error distribution & 11.90 & 1.90 \\
\hline Holt Winter point forecast computation coupled with a Garch error distribution & 18.08 & 2.21 \\
\hline Holt Winter point forecast computation coupled with a BootStrap error distribution & 30.11 & 7.86 \\
\hline Persistent point forecast computation coupled with a Gaussian error distribution & 72.16 & 0.20 \\
\hline
\end{tabular}




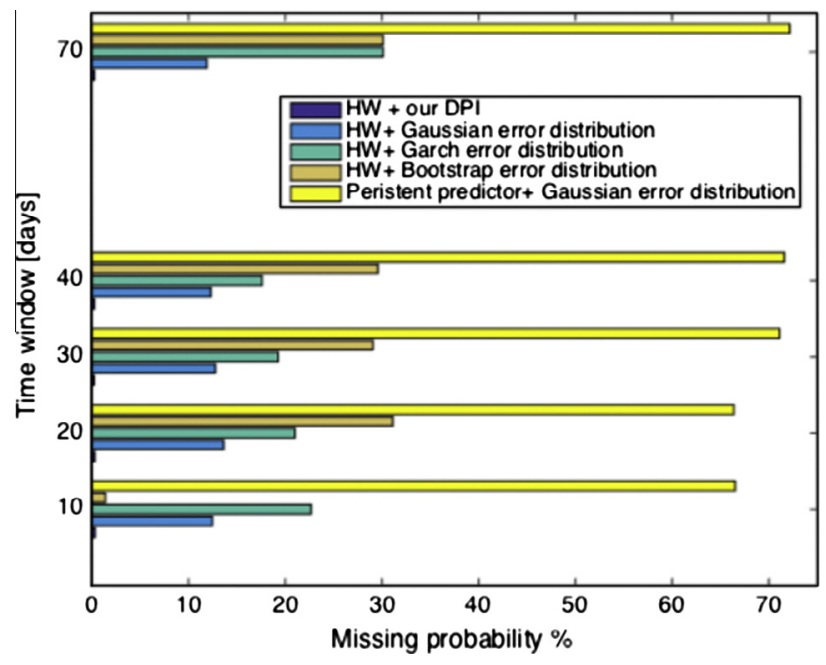

Fig. 8. Graphical comparison of different PIs in term of missing probability (time-steps of $250 \mathrm{~ms}$ ).

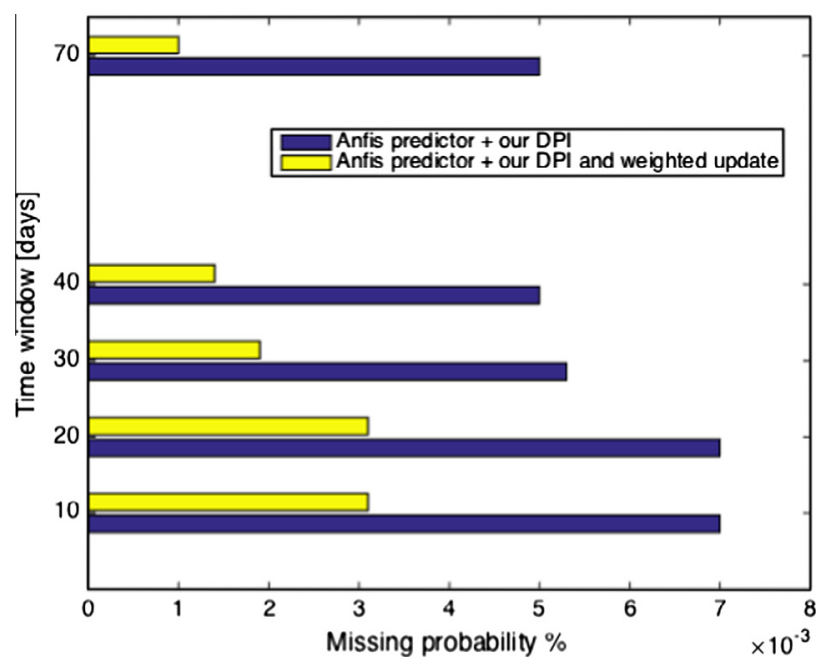

Fig. 9. Graphical comparison of ANFIS point forecast computation coupled with our DPI. Comparison of step-by-step update and weighted update with a time horizon of $2 \mathrm{~s} \mathrm{w}$.

- R-update method: method \#1 enables taking into account instantaneously any new dynamics associated with the solar irradiance. For the R-update with method $\# 1$, the higher the width of the learning time window, the lower the value of $\Gamma$ and $X_{\mathrm{IN}}$. Method \#3 is the most accurate: it shows the importance of giving a higher weight to the latest dynamics. For method \#2 the above observations do not hold.

Figs. 4-6 illustrate a graphical comparison of the effects of the correlation matrix update methods on the value of the missing probability, for the three time-steps $(250 \mathrm{~ms}$, $500 \mathrm{~ms}, 750 \mathrm{~ms}$ ). These figures illustrate the results summarized in Table 1.

Table 2 provides the value of the considered performance metrics, in terms of $\Gamma$ and $X_{I N}$, for method \#3

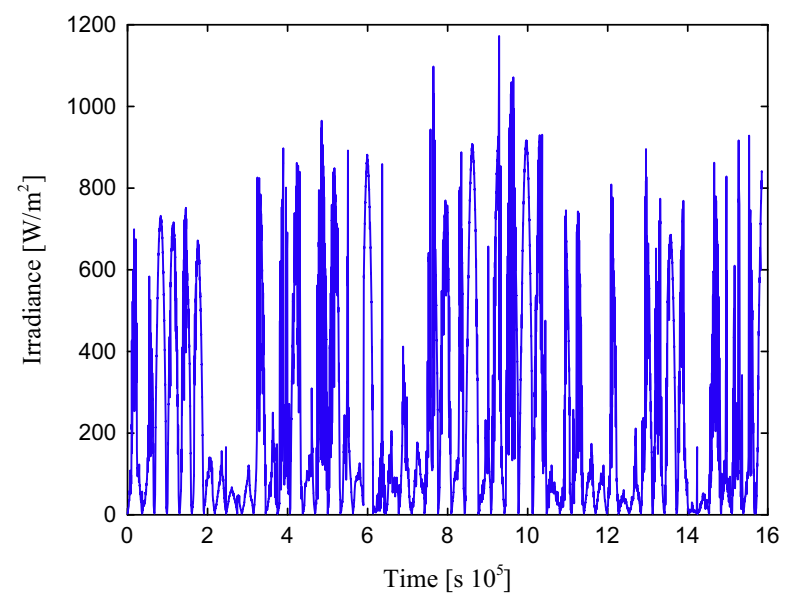

Fig. 10. Irradiance time evolution during 70 days of measurements.

weight $w_{2}$ for different values of the confidence-interval. It is possible to quantify the increase of the percentage of points outside the bounds for low confidence intervals and the corresponding wider PIs.

Fig. 7 illustrates graphically the results reported in Table 2.

\subsection{Comparison with the other prediction interval methods}

In this subsection we compare our DIP with the conventional methods discussed in Section 3. We consider the following cases:

(a) HW forecast method with the proposed DIP.

(b) HW forecast method with prediction-interval based on Eq. (2), specifically the variance computation with Gaussian distribution of the point-forecast error.

(c) HW forecast method with prediction interval based on (3), specifically the variance computation with GARCH $(1,1)$ model distribution of the point forecast error.

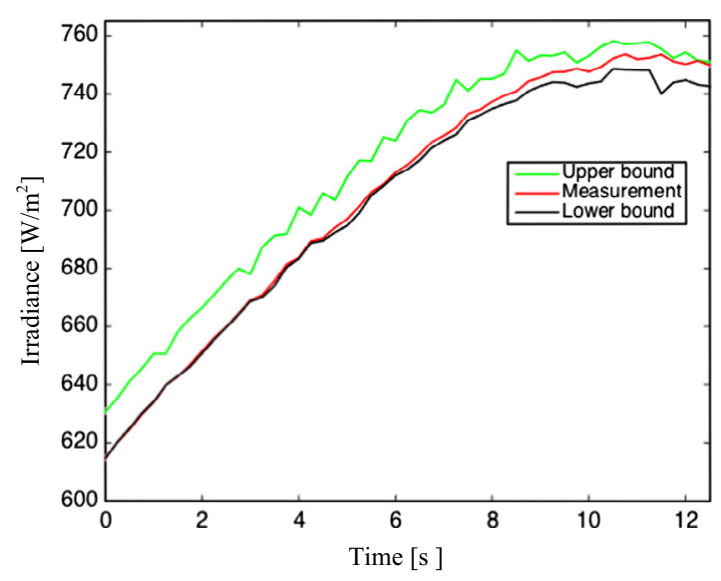

Fig. 11. Example of the time evolution of DIP during high dynamics. 
Table 4

Performance of DPI when the point predictor is provided by the adaptive neuro fuzzy inference system. Time step $=250 \mathrm{~ms}$.

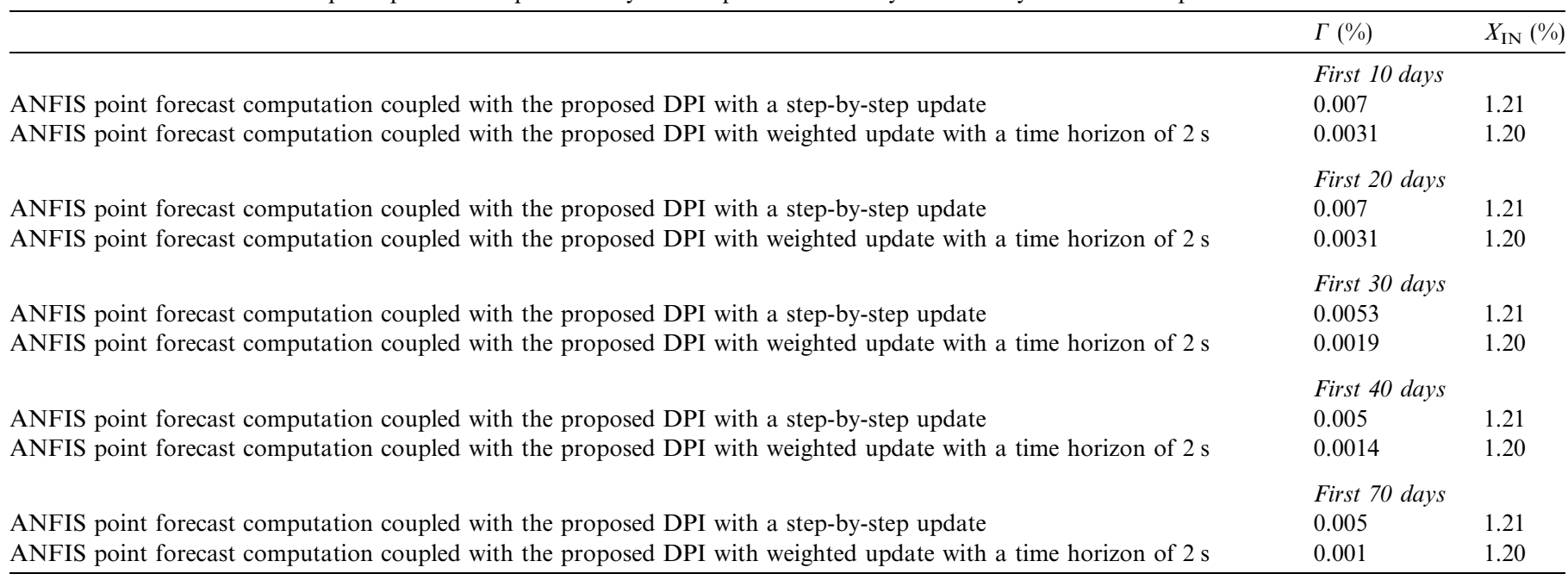

(d) HW forecast method with prediction-interval based on Eq. (4), specifically the Bootstrap distribution of the point-forecast error.

(e) Persistent forecast method and prediction interval based on Eq. (2).

We use a time-step of $250 \mathrm{~ms}$ as it provides the best results. For the proposed DIP we use the $\mathbf{R}$ matrix update method \#1 as it is the only one that can be fairly compared with the others.

We perform the comparison for the same data as in Table 1. The error distribution of cases (b), (c), and (d) are also updated at each sample. The main results of this analysis are shown in Table 3. The following considerations can be derived (see Fig. 8).

- The DIP shows the best performances in terms of trade of between $\Gamma$ and $X_{\mathrm{IN}}$. Our DIP has a $\Gamma$ metric one order of magnitude lower, with respect to some other PIs.

- For the two error distributions (the Gaussian and the GARCH), the larger the amount of data for the learning process is, the higher the accuracy of the predictor is.

- The Bootstrap predictor improves its accuracy when large amounts of data are accounted. In this respect, during the first 10 days, it performs as the proposed DIP as no extreme irradiance dynamics were observed.

- The persistent predictor does not show any remarkable improvement and the lower value of $\Gamma$ is largely worsened by the higher values of $X_{\mathrm{IN}}$, i.e. it produces prediction intervals that are too wide.

Using the above considerations, we can conclude that our DIP clearly shows the best performances in terms of combined metrics $\Gamma$ and $X_{\mathrm{IN}}$. It is worth observing that the DIP coupled with the update method \#3 has the best value of $\Gamma$, even one order of magnitude lower than the persistent model coupled with a Gaussian errordistribution.
The results summarized in Table 3 are shown in Fig. 8 .

For sake of completeness, Fig. 11 illustrates the time evolution of the solar irradiance along with the predicted interval with the DIP during a typical dynamic.

\subsection{Adaptive neuro fuzzy inference system}

In order to highlight the independence of the DIP from the model used for the point forecast, we applied the DIP to a specific forecast technique that does not provide any information on the prediction intervals. For this purpose, we selected the adaptive neuro fuzzy inference system (ANFIS) with a Gaussian function used as the mother function. For this purpose, we adopt a toolbox available in the Matlab $^{\circledR}$ programming environment. ${ }^{2}$ Table 4 illustrates the summary of the performances of our DIP coupled with the ANFIS point predictor. The update methods are methods \#1 and \#3 with weight $w_{2}$ and the time-step is $250 \mathrm{~ms}$.

Fig. 9 summaries the result shown in Table 4. In Table 4, we observe that the DIP is robust because the $\Gamma$ metric reduces its value for a larger learning time-window and the metric $X_{\text {IN }}$ keeps reasonable values. Comparing with the corresponding values in Table 1, we find that the combination of ANFIS with the proposed DIP provides a smaller miss probability $(\Gamma)$ with the same prediction interval width $\left(X_{\mathrm{IN}}\right)$.

\footnotetext{
${ }^{2}$ An adaptive neuro-fuzzy inference system (or adaptive network-based fuzzy inference system - ANFIS) is an artificial neural network based on the Takagi-Sugeno fuzzy inference system. The technique integrates both neural networks and fuzzy logic principles and, consequently, it has the advantage to capture in a single framework the benefits of both neural network and fuzzy systems. Its inference corresponds to a set of fuzzy IFTHEN rules can approximate nonlinear functions. In the work here proposed we used the ANFIS model to compute the point forecast value of the solar irradiance. Then, the results associated with this point prediction, are used to create and update the correlation matrix $\mathbf{R}$ of our DPI.
} 


\section{Conclusions}

In this paper we discussed the problem of the ultrashort-term prediction of the solar irradiance. We focused our attention on proposing a dynamic interval predictor (DIP) that does not require a specific point-prediction method or any hypothesis on the error-forecast distribution. The proposed DIP relies on an on-line assessment of the statistical correlations between the solar irradiance time derivative and the point-forecast error in the next forecasting time-step. The main features of the proposed DIP are here summarized:

- The DIP does not depend directly on the method used for the point forecast; in particular, it can work with methods such as artificial neural networks that do not provide prediction interval.

- It is able to take into account high dynamics.

- It improves its performance during its use because it is able to correct the magnitude of the predictions intervals for future computations.

- It can produce results for any required confidence level.

We have compared our DIP with other methods for prediction intervals that use a specific error-distribution (i.e., Gaussian, GARCH and Bootstrap). We made such an assessment by using an experimentally obtained time series of solar irradiance of 70 days. The main results of this comparison show that the DIP exhibits the best performance in terms of tradeoff between miss probability and interval width.

We would like to conclude this section by underlining that the rationale to develop such a DIP is associated with its potential use in real-time optimal control-processes of electrical distribution networks characterized by a large penetration of photovoltaic power plants. Indeed, perspective real-time optimal control processes of these grids might take advantage of the availability of our DIP.

\section{References}

Bacher, P., Madsen, P.H., Nielsen, H.A., 2009. Online short-term solar power forecasting. Sol. Energy 83, 1772-1783.

Behrang, M.A., Assareh, E., Ghanbarzadeh, A., Noghrehabadi, A.R., 2010. The potential of different artificial neural network (ANN) techniques in daily global solar radiation modeling based on meteorological data. Sol. Energy 84, 1468-1480.

Bernstein, A., Reyes Chamorro, L.E., Le Boudec, J.-Y., Paolone, M., 2015. A composable method for real-time control of active distribution networks with explicit power setpoints. Part I: framework. Electr. Power Syst. Res. 125, 254-264.
Brockwell, P.J., Davis, Richard A., 2002. Introduction to Time Series and Forecasting. Taylor and Francis.

Heniche, A., Kamwa, I., Cauchon, L., 2013. Multiagent stochastic simulation of minute-to-minute grid operations and control to integrate wind generation under AC power flow constraints. IEEE Trans. Sustain. Energy 4, 619-629.

Hindammn, R., Koelher, B., Ord, J., Snyder, R., 2008. Forecasting with Exponential Smoothing. Springer.

Kardakos, E.G., Alexiadis, M.C., Vagropoulos, S., Simoglou, C.K., Biskas, P.N., Bakirtzis, A.G., 2013. Application of time series and artificial neural network models in short-term forecasting of $\mathrm{PV}$ power generation. In: Proceeding of 48th power engineering conference, Dublin, Ireland, pp. 1-6.

Le Boudec, J.Y., 2010. Performance Evaluation of Computer and Communication Systems. EPFL CRC Press.

Lorenz, E., Hurka, J., Heinemann, D., Beyer, H.G., 2009. HG, irradiance forecasting for the power prediction of grid connected photovoltaic systems. IEEE J. Selected Topics Appl. Earth Observations Remote Sensing 2, 2-10.

Marquez, R., Coimbra, C.F.M., 2011. Forecasting of global and direct solar irradiance using stochastic learning methods, ground experiments and the NWS database. Sol Energy 85, 746-756.

Mellit, A., Kalogirou, A.S., 2008. Artificial intelligence techniques for photovoltaic applications: a review. Prog. Energy Combust. Sci. 34, 574-632.

Mellit, A., Pavan, A.M., 2010. A 24-h forecast of solar irradiance using artificial neural network: application for performance prediction of a grid-connected PV plant at Trieste, Italy. Sol. Energy 84, 807-821.

Pinson, P., Tastu, J., 2014. Discussion of prediction intervals for shortterm wind farm generation forecasts and combined nonparametric prediction intervals for wind power generation. IEEE Trans Sustain. Energy 5, 1019-1020.

Reyes Chamorro, L.E., Bernstein, A., Le Boudec, J.-Y., Paolone, M., 2015. A composable method for real-time control of active distributionnetworks with explicit power setpoints. Part II: implementation and validation. Electr. Power Syst. Res. 125, 265-280.

Segura, J.V., Vercher, E., 2001. A spreadsheet modeling approach to the Holt-Winters optimal forecasting. J. Operational Res. 131 (2), 375-388.

Sfetsos, A., Coonick, A.H., 2000. Univariate and multivariate forecasting of hourly solar radiation with artificial intelligence techniques. Sol. Energy 68, 169-178.

Singh, V.P., Vijay, V., Bhatt, M.S., Chaturvedi, D.K., 2013. Generalized neural network methodology for short term solar power forecasting. In: Proceedings of 13th international conference on environment and electrical engineering, Rome, Italy, pp. 58-62.

Song, I., Yun, S.-Y., Kwon, S., Kwak, N., 2013. Design of smart distribution management system for obtaining real-time security analysis and predictive operation in Korea. IEEE Trans. Smart Grid 4, 375-382.

Trapero, J.R., Martin, A., Garcia, F.P., 2014. Short-term solar irradiance forecasting based on holt-winters exponential smoothing. In: Proceeding of 26th European conference on operational research, Rome, Italy.

Vrakopoulou, M., Margellos, K., Lygeros, J., Andersson, G., 2013. A probabilistic framework for reserve scheduling and N-1 security assessment of systems with high wind power penetration. IEEE Trans. Power Syst. 28, 3885-3896.

Wan, C., Xu, Z., Pinson, P., Yang Dong, Z., Wong, K.P., 2014. Optimal prediction intervals of wind power generation. IEEE Trans. Power Syst. 29, 1166-1174. 